\title{
Superradiance of Aggregated Thiacarbocyanine Molecules
}

\author{
Serdar Özçelik \\ Chemistry Department, Bilkent University, 06533-Ankara, Turkey
}

\author{
Daniel L. Akins* \\ Center for Analysis of Structures and Interfaces (CASI), Department of Chemistry, The City College of \\ The City University of New York, New York, New York 10031
}

Received: May 19, 1999; In Final Form: August 16, 1999

\begin{abstract}
Emission lifetime and steady-state spectral measurements of aggregated and monomeric 3,3'-diethyl-5,5'dichloro-9-phenylthiacarbocyanine (DDPT) in methanol and aqueous solutions at room temperature have been conducted. We deduce that in aqueous solution DDPT molecules that maintain their monomer emission lifetime and spectral properties exist intimately with molecules that are coherently interconnected in the excitonic structure. Simultaneous superradiance and monomer fluorescence from DDPT molecules occur and allows for a direct comparison of superradiance emission properties of aggregated molecules to the emission properties of the single molecule. We have found that the fluorescence lifetime of the aggregate is excitation wavelength dependent, and are led to conclude that superradiance by aggregated molecules and energy transfer from monomeric species to the aggregate play important roles in determining the optical dynamics of the aggregated system. We also report some of the photophysical properties of DDPT.
\end{abstract}

\section{Introduction}

Research dealing with exciton dynamics within molecular aggregated structures that are formed by noncovalent, selfassembly of molecules has become extremely active-principally because of the interest in exploiting molecular structures for optical information processing and device applications. The inherent participation of the quantum states of molecules in defining the state-energies of such aggregated structures provides a means for control of both the energies of the composite structure and the dynamics of energy flow, upon excitation, within the structure.

Phenomena such as superradiant emission, nonlinear optical response, exciton-exciton annihilation and exciton-phonon interactions have been specific research foci for many molecular aggregate systems. These phenomena represent system parameters that might be utilized to develop new materials with unique photonic and optoelectronic properties.

The focus of this manuscript is on superradiance from a cyanine dye system that simultaneously evinces monomer and aggregate emissions, allowing for a more direct comparison of superradiance characteristics relative to those of the monomer.

Superradiance (viz., coherent spontaneous emission (CSE)), it might be noted, is a somewhat difficult concept to comprehend in terms of the structural geometry of the aggregate and the influence of structure on the cooperative emission rate, though several studies have provided clear evidence of the basic phenomenon. Systems in which superradiance has been verified include 1,1'-diethyl-2,2'-cyanine (PIC) in ethylene glycol/water glass at low temperature, ${ }^{1,2} \mathrm{PIC}$ adsorbed onto an $\mathrm{AgBr}$ surface at room temperature, ${ }^{3}$ 1, $1^{\prime}$-diethyl-3,3'-di(3-sulfopropyl)-5, 5',6,6'tetrachlorobenzimidazolocarbocyanine (i.e., BIC) in ethylene glycol/water glass at low temperature; ${ }^{4}$ and, recently from this laboratory, 1,1',3,3'-tetraethyl-5,5',6,6'-tetrachlorobenzimid-

* Corresponding author. azolocarbocyanine (TTBC) and BIC adsorbed at room temperature onto colloidal silica and silver particles, respectively. ${ }^{5,6}$

Implicit in the phenomenon of superradiance is the concept of a coherence size for the emitting entity, ${ }^{7-9}$ often considered a localized region of the physical aggregate structure. The latter may be comprised of an enormous number of monomeric species, while the superradiant emitting entity is assumed to be composed of a small number of molecules that collectively and coherently emit radiation. The coherence size specification is used to designate the number of molecules in the superradiant entity, and the radiative decay probability of the aggregate, in the simplest case, is related to the radiative decay probability of the incorporated monomeric species by a multiplicative factor corresponding to the coherence size. The issue of coherence size may also arise when absorption and scattering of radiation by aggregated structures are of interest.

For the present study we have conducted steady-state fluorescence and time-resolved fluorescence lifetime measurements for 3,3'-diethyl-5,5'-dichloro-9-phenylthiacarbocyanine (referred to hereinafter as DDPT; see insert in Figure 1 for structure) in methanol (where the monomeric species exists) and in aqueous homogeneous solution (where the aggregate predominates, though monomer is still present). We have also determined some of the photophysical properties of DDPT for the same solution systems for which lifetime measurements were made. We have deduced that superradiance plays a crucial role in optical dynamics of the aggregated system and energy transfer from monomer to aggregate results in longer fluorescence lifetime for the aggregate.

\section{Experimental Section}

All spectroscopic measurements were conducted at room temperature. DDPT was purchased from the Japanese Research Institute for Photosensitizing Dyes, Ltd., Okayama, Japan, and used without further purification. 


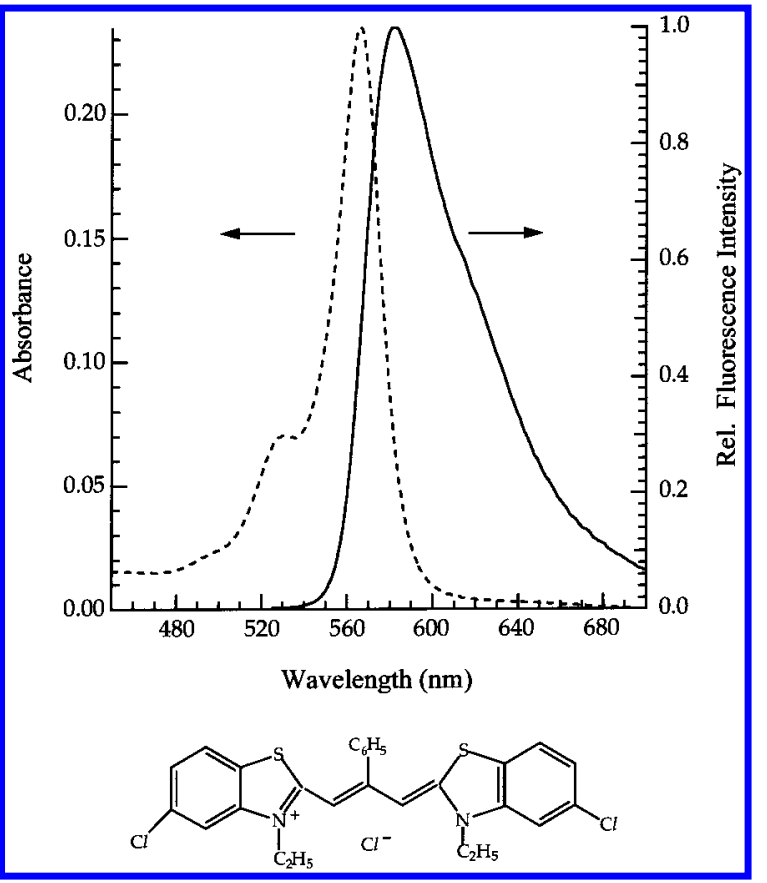

Figure 1. Absorption and fluorescence spectra of DDPT in methanol at ca. $10^{-5} \mathrm{M}$ concentration in a $0.1-\mathrm{mm}$ cuvette. Insert shows the chemical structure of DDPT with chloride counterion.

Absorption spectra were recorded using a Perkin-Elmer Lambda 19, UV-vis/NIR spectrometer. Steady-state fluorescence spectra were acquired using a SPEX Fluorolog- $\tau 2$ spectrofluorimeter. Time dependent spectral emission measurements utilized a Hamamatsu streak camera (Model C4334) and optically coupled charge-coupled-device (CCD) array detector combination, positioned at the exit plane of a Chromex 250i imaging spectrometer. The limiting time resolution for the system, as indicated by the instrument response to the incident laser pulse of. 10 ps (see below), is ca. 30 ps, as shown in Figure 3 below.

The excitation light source was a Coherent 702 mode-locked, picosecond dye laser that was pumped by a Coherent Antares 76s Nd:YAG laser. The excitation wavelengths used for the aggregate were 575 and $615 \mathrm{~nm}$, the latter near the J-aggregate absorption maximum (see Figure 2, below). The excitation wavelength for monomer in methanol and in aqueous homogeneous solution was $575 \mathrm{~nm}$. This latter wavelength is near the absorption maximum of the monomer (see Figure 1, above).

To prepare J-aggregated DDPT, $0.4 \mathrm{~mL}$ of $4.0 \mathrm{mM}$ DDPT in methanol was mixed with $135.6 \mathrm{~mL}$ of $0.1 \mathrm{M} \mathrm{KCl}$ aqueous solution. The samples were excited with pulses of energy of ca. $0.13 \mathrm{~nJ} /$ pulse and of $10 \mathrm{ps}$ duration, with repetition rate of $76 \mathrm{MHz}$. The intensity of the incident radiation was adjusted through the use of calibrated neutral density filters and was kept below $10^{11}$ photons $/ \mathrm{cm}^{2}$ in order to minimize lifetime shortening effects attributable to exciton-exciton annihilation. ${ }^{10}$ Also, frontsurface luminescence measurements, using a $0.3 \mathrm{~mm}$ single optical fiber to collect emission, were conducted (see below), and the sample was flowed through the excitation region to minimize photodecomposition of the sample.

\section{Results and Discussion}

Figure 1 shows absorption and emission spectra of monomeric DDPT in methanol. The absorption spectrum has two main bands at ca. 566 and $528 \mathrm{~nm}$, attributable to the $0 \longleftarrow 0$ and $1 \longleftarrow 0$ vibronic transitions, respectively. The emission spectrum is redshifted with respect to the absorption spectrum and has a shape

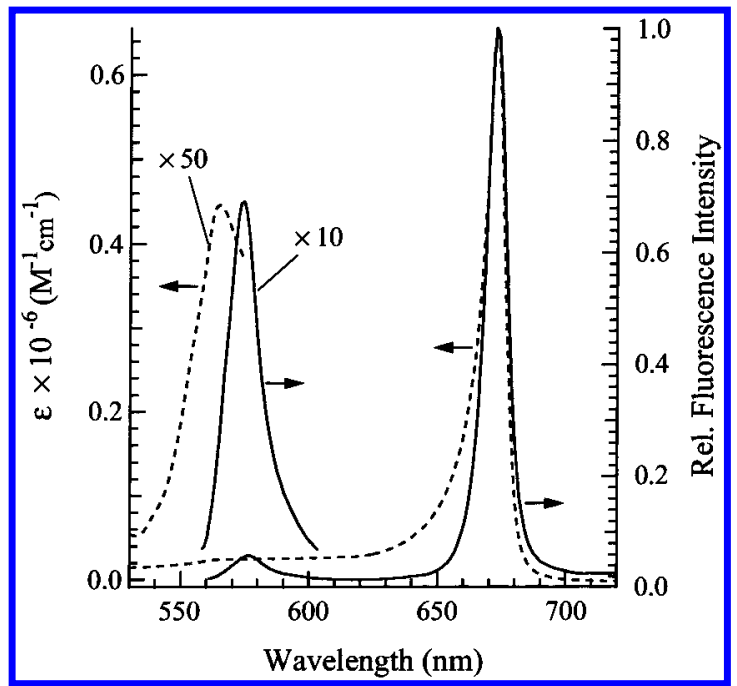

Figure 2. Absorption and fluorescence spectra of $10^{-5} \mathrm{M}$ DDPT in water. Scale factors for enhancements of signals for weak bands are indicated as numbers tagged to bands.

suggestive of the mirror-image structure that is expected of most molecules. The emission has a peak at ca. $582 \mathrm{~nm}$.

Figure 2 shows absorption and emission spectra of DDPT in aqueous solution, in which DDPT exists predominantly in an aggregated form. The absorption spectrum has two bands, an intense band at ca. $673 \mathrm{~nm}$ and a much weaker one at $566 \mathrm{~nm}$. The band at $673 \mathrm{~nm}$ is attributable to the J-aggregate, while the band at $566 \mathrm{~nm}$, corresponding to a separation from the $673 \mathrm{~nm}$ band of some $2809 \mathrm{~cm}^{-1}$-unlikely to be due to a vibronic absorption band of the aggregate-is attributable to absorption by the monomer. The emission spectrum in Figure 2 (excited with $560 \mathrm{~nm}$ radiation) likewise shows two bands (at 673 and $578 \mathrm{~nm}$; the narrow emission at ca. $673 \mathrm{~nm}$ is attributable to J-aggregate, and essentially is in resonance with the J-aggregate absorption, while the emission band at $578 \mathrm{~nm}$ (consistent with the red-shift expected between absorption and fluorescence bands) is attributed to monomeric fluorophores). It is to be noted that the simultaneous presence of both monomer and aggregate emissions allows for the determination of the relative fluorescence efficiency of J-aggregated DDPT, since monomers exist in the same microenvironment as the aggregate.

The nature of the putative monomer in an environment in which aggregates predominates is of interest. An obvious possibility is that two types of monomers are involved in the physical aggregate; one type, though intercalated in the aggregate structure is essentially isolated and differs from another type that is coherently coupled to other monomers and collectively form the superradiant emitter. Another possibility is that there might be free monomers in solution that exist separately from those that are involved in the physical aggregate. An aid in assessing whether one or both of the possible monomer/aggregate pictures apply is provided by a determination of the direction of spectral shift of monomer features in the aggregate system when compared to free monomer at low concentration in an organic solvent. It is expected that the monomer when intercalated within the aggregate structure would experience a somewhat more rigid environment than do free monomers, whether in aqueous solution or in $\mathrm{MeOH}$ (the organic solvent we have used in this work). We have found, in fact (as mentioned above), that the monomer in $\mathrm{MeOH}$ absorbs at $566 \mathrm{~nm}$ and emits at $582 \mathrm{~nm}$, while the monomer in the aggregate environment absorbs at $566 \mathrm{~nm}$ and emits at $578 \mathrm{~nm}$. Hence, a blue shift of the monomer's emission occurs, sug- 
gesting that monomers experience a constricting environment, such as would be expected if they were intercalated within an aggregate structure. This picture is also consistent with earlier findings from this laboratory regarding $2,2^{\prime}$-cyanine (viz., PIC) and $4,4^{\prime}$-cyanine. ${ }^{11,12} \mathrm{We}$ ascertained from Raman scattering studies of both 2,2'-cyanine and 4,4'-cyanine adsorbed onto smooth, polished surfaces that the Raman spectra consist of bands due to adsorbed J-aggregate as well as effectively monomeric molecules. In the case of 2,2'-cyanine-as revealed from $\mathrm{pH}$-dependent Raman frequencies with $488 \mathrm{~nm}$ excitationwe were able to correlate numerous Raman bands of the adsorbate with those of the crystalline material. ${ }^{11}$ We further found that Raman overtone and combination bands for both adsorbates could be excited by pumping the respective monomer's adsorption frequency, ${ }^{11,12}$ and such bands (incidentally) could be used in determining the common parentage (e.g., origin due to protonated or nonprotonated species) of various fundamental bands. ${ }^{11,12}$

In the present work, we have also determined the relative room-temperature fluorescence efficiency of aggregated DDPT. Fluorescence spectra were taken using front-face excitation with a $0.1-\mathrm{mm}$ cuvette. Low sample concentrations (near $10^{-5} \mathrm{M}$ ) were used to eliminate self-absorption, which may result in radiation trapping between excited-state and ground-state aggregates, since the absorption and emission spectra of the J-aggregate overlap almost completely with nearly equal bandwidths.

To determine the fluorescence efficiency, fluorescence emissions of aggregated and monomeric DDPT were excited using $560-\mathrm{nm}$ radiation. The respective bands were integrated with respect to wavelength and divided by the absorbance at 560 $\mathrm{nm}$. This approach was also used for the fluorescence band of the aggregate obtained with 615-nm excitation; an additional correction factor was used to correct for the absorbance difference at $615 \mathrm{~nm}$. The monomer's integrated fluorescence divided by the absorbance at 560-nm in the aggregate environment was used as reference and it was taken as 1.0. As a result of this procedure, the fluorescence efficiency of the aggregate for 560-nm excitation was found to be 10-fold increased compared to the monomer in the aggregate environment. For the 615-nm excitation, the fluorescence efficiency of the aggregate was enhanced 22-fold. We interpret these results as evidence for superradiance from J-aggregates of DDPT at room temperature. These data are summarized in Table 1.

To confirm superradiant enhancement, fluorescence lifetime measurements were also carried out for the J-aggregate and monomer in $\mathrm{MeOH}$ and in the aqueous system in which aggregation predominates. The putative monomers in $\mathrm{MeOH}$ and aqueous solutions were excited at $575 \mathrm{~nm}$, while Jaggregates were excited at both 575 and $615 \mathrm{~nm}$. Figure 3 shows typical fluorescence decays using our streak-camera system of the J-aggregate excited at 575 and $615 \mathrm{~nm}$, and the monomer in the aggregate environment, excited at $575 \mathrm{~nm}$.

The fluorescence lifetime of the aggregate was found to change with excitation wavelength (see Table 1). The fluorescence lifetime of the aggregate was found to be $64.5 \mathrm{ps}$ for excitation at $575 \mathrm{~nm}$, while the lifetime found using 615-nm excitation was 25.3 ps. The similar magnitude of the fluorescence lifetimes of the aggregate and monomer when $575 \mathrm{~nm}$ excitation was used can be rationalized as the creation of a higher excited state (in the present case, the excited monomer) that subsequently transfers energy (incoherently and with loss channels) to the aggregate, thus prolonging the aggregate's lifetime. The shorter lifetime with direct excitation of the
TABLE 1: Photophysical Parameters of Monomeric DDPT in $\mathrm{MeOH}$ and Aqueous Solutions ( $\mathrm{M}$ and $\mathrm{M}^{\prime}$, respectively) and J-aggregate in Aqueous Solution $(J)$ at Room Temperature

\begin{tabular}{lccc}
\hline \multicolumn{1}{c}{ parameters } & \multicolumn{1}{c}{$\mathrm{M}$} & $\mathrm{M}^{\prime}$ & $\mathrm{J}$ \\
\hline$\lambda_{\text {abs }}(\mathrm{nm})^{a}$ & 566 & 566 & 673 \\
$\Delta v_{\text {abs }}\left(\mathrm{cm}^{-1}\right)^{b}$ & 650 & & 178 \\
$\lambda_{\text {fluo }}(\mathrm{nm})^{c}$ & 582 & 578 & 673 \\
$\Delta v_{\text {fluo }}\left(\mathrm{cm}^{-1}\right)^{d}$ & 720 & & 168 \\
$\Delta v_{\text {Stokes }}\left(\mathrm{cm}^{-1}\right)$ & 485 & 366 & 0 \\
$\phi_{\text {fluo }}(575 \mathrm{~nm})$ & $0.015 \pm 0.002^{e}$ & $1.0^{f}$ & 10 \\
$\quad(615 \mathrm{~nm})$ & & & 22 \\
$\tau_{\text {fluo }}(\mathrm{ps})(575 \mathrm{~nm})$ & $71.9 \pm 0.7^{g}$ & $70.8 \pm 0.4^{g}$ & $64.5 \pm 0.4^{h}$ \\
$\quad(615 \mathrm{~nm})$ & & & $25.3 \pm 0.5^{i}$ \\
$k_{\mathrm{r}} \times 10^{8}\left(\mathrm{~s}^{-1}\right)$ & $2.0 \pm 0.4$ & & \\
$k_{\mathrm{nr}} \times 10^{10}\left(\mathrm{~s}^{-1}\right)$ & $1.3 \pm 0.1$ & & \\
$\epsilon \times 10^{5}\left(\mathrm{M}^{-1} \mathrm{~cm}^{-1}\right)$ & $3.0 \pm 0.1$ & & \\
$k_{\mathrm{J}} / k_{\mathrm{M}}(575 \mathrm{~nm})$ & & & $\sim 11$ \\
$\quad(615 \mathrm{~nm})$ & & & $\sim 62$ \\
$\quad$ & & &
\end{tabular}

${ }^{a}$ Absorption spectra maxima. ${ }^{b}$ The bandwidth of the $0 \leftarrow 0$ lowest excited electronic state transition is obtained by decomposing absorption spectra. ${ }^{c}$ Fluorescence spectra maxima. ${ }^{d}$ The bandwidth is obtained by spectrally decomposing fluorescence spectra. ${ }^{e}$ Absorbance values were less than 0.06 for the samples and reference, rhodamine $6 \mathrm{G}$ in EtOH. ${ }^{f}$ Taken as reference and set equal to $1.0{ }^{g}{ }^{g}$ Single exponential decays with $\chi^{2}$ varying between 1.1 and 1.4. ${ }^{h}$ A double exponential decay with shorter lifetime 64.5 ps (95\%) and longer lifetime of 335 ps $(2 \%)$ duration; $\chi^{2}=1.44 .{ }^{i}$ A triple exponential decay with shorter lifetime of $25.3 \mathrm{ps}(97.7 \%)$, second of 86.9 ps $(2.2 \%)$ duration, and third of 510 ps $(0.1 \%)$ duration; $\chi^{2}=1.3$.

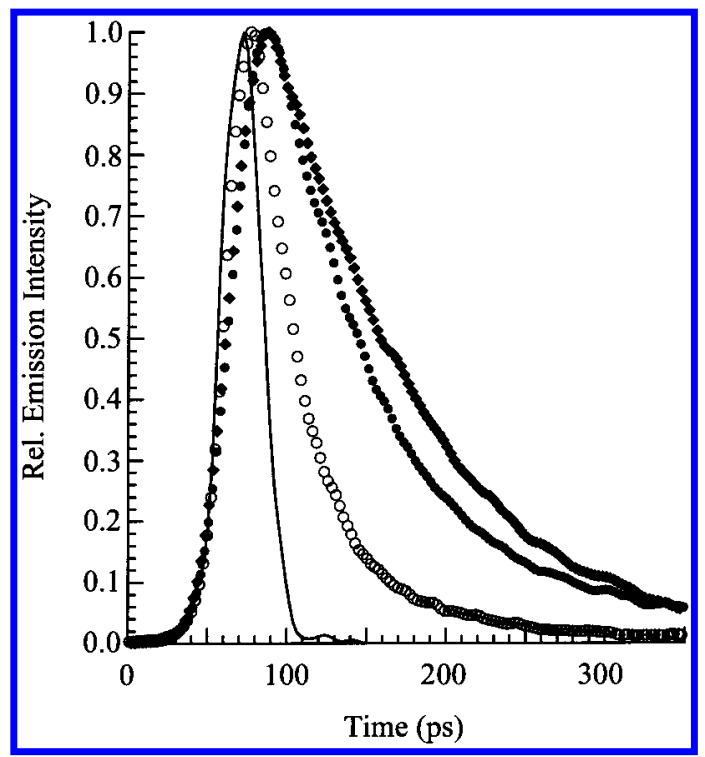

Figure 3. Fluorescence decay of DDPT in aqueous solution for J-aggregate band excited at $575 \mathrm{~nm}(\bullet)$ and $615 \mathrm{~nm}(\mathrm{O})$, and monomer band excited at $575 \mathrm{~nm}(\bullet)$. Laser pulse excitation was of ca. 10-ps duration and a streak-camera (see text) was used to capture decay. The $\mathrm{J}$-aggregate fluorescence decay for $575-\mathrm{nm}$ excitation utilized a detection band-pass ranging from 650 to $750 \mathrm{~nm}$, and was centered on the aggregate emission peak at $673 \mathrm{~nm}$. The J-aggregate fluorescence decay for 615-nm excitation utilized a detection band-pass ranging from 640 to $715 \mathrm{~nm}$, and was centered on the aggregate emission peak at $673 \mathrm{~nm}$. For the monomer, the excitation frequency was $575 \mathrm{~nm}$ and the detection range was 585 to $650 \mathrm{~nm}$, with the emission centered at $580 \mathrm{~nm}$. The solid line represents the temporal response of the detection system to the incident laser excitation.

aggregate (with 615-nm radiation) in this picture would derive from the elimination of the rate-limiting energy transfer (or relaxation) step as well as avoidance of energy depletion pathways that occasion the indirect process. A similar finding was reached by us in a study of a water-soluble porphyrin aggregate, whose excitonic Q-state when indirectly excited by 
relaxation from the porphyrin aggregate's singlet $\mathrm{S}$ (Soret) energy state has a longer lifetime than when excited directly to the excitonic Q-band. ${ }^{13}$ The explanation that we have provided is that nonradiative pathways in the relaxation process lead to a diminished coherence length and a resultant longer fluorescence lifetime when compared to an aggregate formed directly through absorption of radiation. ${ }^{13}$

In the present case, the energy transfer from the monomer to the aggregate would also be expected to be less than $100 \%$ efficient and to result in a smaller coherence length and thus longer lifetime. But another way of explaining the increased lifetime would be to think of the singlet excited state of the monomers as a "bottle-neck" state, since the lifetime of the monomer is longer than that of the aggregate, which would slow the emission process, prolonging the effective lifetime of the emission.

In any case, the lifetime upon direct excitation of the aggregate absorption through the use of $615 \mathrm{~nm}$ radiation is taken by us as the true measure of the aggregate's emission rate, unimpeded by rate-limiting steps.

Table 1 also provides the relative radiative rate constant $\left(k_{\mathrm{J}}\right)$ of aggregated DDPT, as estimated from the relationship

$$
\frac{k_{\mathrm{J}}}{k_{\mathrm{M}}}=\frac{\phi_{\mathrm{J}} \tau_{\mathrm{M}}}{\phi_{\mathrm{M}} \tau_{\mathrm{J}}}
$$

where $k_{\mathrm{J}}$ and $k_{\mathrm{M}}$ are the relative radiative rate constants of the aggregate and monomer, respectively; $\phi_{\mathrm{J}}$ and $\phi_{\mathrm{M}}$ are the relative fluorescence efficiencies of aggregate and monomer, respectively; and $\tau_{\mathrm{J}}$ and $\tau_{\mathrm{M}}$ are, respectively, the fluorescence lifetimes of the aggregate and monomer in the aggregate environment. The ratio $k_{\mathrm{J}} / k_{\mathrm{M}}$ is expected to be proportional to the number of the molecules involved in the coherent decay of the J-aggregate. This number is found to be ca. 62 , in the present case, for direct excitation of the aggregate with 615-nm radiation, and ca. 11 for indirect excitation of the aggregate using 575-nm radiation. These values are indicative of superradiance from the aggregate and are consistent with the view that direct excitation is a more efficient process.

More quantitative efforts at determining the rate constant for the conversion of excited monomer energy into excitonic energy of the aggregate will be reported in an upcoming paper from this laboratory dealing with optical and population dynamics for DDPT. In this latter study time-dependent emission is measured, using the streak-camera described in the present manuscript, and decomposed with 10 ps resolution into component emissions attributed to transient states.

\section{Conclusion}

Steady-state spectral and time-resolved lifetime measurements for monomeric and aggregated DDPT have been conducted and are interpreted as supporting the concept of room-temperature superradiance by the aggregate. Systems involving monomeric DDPT in MeOH and aggregated DDPT in aqueous solution have been studied using selected excitation frequencies. It is deduced that when monomer and aggregate steady-state absorption/ emission spectral features exist at the same time, unincorporated monomers entities -i.e., monomer that is not coherently coupled to other monomers in the aggregate but is spatially constricted by being incorporated in the physical aggregate-exist as part of the physical aggregate.

Fluorescence quantum efficiencies of the aggregate (at two different excitation wavelengths) relative to the monomer are determined, as are lifetimes and other photophysical parameters for DDPT. An important determination is that energy transfer from monomer to aggregate occurs and results in a longer fluorescence lifetime for the aggregate.

Acknowledgment. This work was supported in part by the CREST Program of the National Science Foundation (NSF) under Grant HRD-9353488, NASA under Grant NAG5-4019 in its FAR program, and the MRSEC Program of the NSF under Award Number DRM-9809687.

\section{References and Notes}

(1) Fidder, H.; Knoester, J.; Wiersma, D. A. Chem. Phvs. Lett. 1990, $171,529$. 6895

(2) Fidder, H.; Terpstra, J.; Wiersma, D. A. J. Chem. Phvs. 1991, 94

(3) Muenter, A. A.; Brumbaugh, D. V.; Apolito, J.; Horn, L. A.; Spano, F. C.; Mukamel, S. J. Phys. Chem. 1992, 96, 2783.

(4) Kamalov, V. F.; Struganova, I. A.; Koyama, Y.; Yoshihara, K. Chem. Phys. Lett. 1994, 226, 136.

(5) Ozçelik, S.; Akins, D. L. Appl. Phvs. Lett. 1997, 71, 3057.

(6) Özçelik, S.; Özçelik, I.; Akins, D. L. Appl. Phys. Lett. 1998, 73 1949.

(7) Grad, J.; Hernandez, G.; Mukamel, S. Phvs. Rev. A. 1988, 37, 3835.

(8) Spano, F. C.; Mukamel, S. J. Chem. Phvs. 1989, 91, 683.

(9) Spano, F. C.; Kuklinski, J. R.; Mukamel, S. J. Chem. Phvs. 1991, 94, 7534.

(10) Sundström, V.; Gillbro, T.; Gadonas, R. A.; Piskarskas, A. J. Chem. Phys. 1988, 89, 2754.

(11) Akins, D. L.; Macklin, J. W. J. Phvs. Chem. 1989, 93, 5999

(12) Akins, D. L.; Macklin, J. W. J. Phys. Chem. 1992, 96, 4515.

(13) Akins, D. L.; Özçelik, S.; Zhu, H.-R.; Guo, C. J. Phvs. Chem. 1996, $100,14390$. 